\title{
Memory scans based on alternative test stimulus representations ${ }^{1}$
}

\author{
ROBERTA L. KLATZKY AND RICHARD C. ATKINSON2 \\ STANFORD UNIVERSITY
}

In a search task similar to that used by Sternberg (1966), Ss were presented with one of three alternative representations of a given stimulus and were required to state whether or not that stimulus was present in a previously memorized list. For each type of stimulus representation and each response (positive or negative), reaction time was recorded. Functions relating reaction time to the size of the memorized list were both qualitatively and quantitatively different from those usually obtained with the Sternberg paradigm. In particular, the form of the functions did not correspond to predictions based on an exhaustive scanning process.

In a series of experiments, Sternberg (1966, 1967a, b, 1968) has used a search paradigm to study the retrieval of information stored in memory. In a typical search task, $S$ sees a memory set of letters, presented in sequence. After a short delay, a warning signal is given, and $S$ is then shown a test stimulus (letter) and required to pull a lever indicating whether or not the test stimulus was a member of the memory set (a positive or negative response, respectively). The dependent variable in this task is response latency, defined as the time between the onset of the test stimulus and S's response. Two reaction-time functions (relating latency to the size of the memory set) are plotted, one for positive and the other for negative responses. These functions serve to indicate the nature of the processes involved in the search task. It is assumed that $S$ stores a representation of the memory set in short-term memory at the beginning of each trial. When he is presented with the test stimulus, he searches through this representation, seeking a match for the stimulus, and the results of this search determine his response. Since $S$ 's responses are required to be virtually error-free, the reaction-time functions may be interpreted as giving accurate information about the search process.

The results of Stemberg's experiments with the task described above have led to several important conclusions. In particular, he has found that the reaction-time functions for both positive and negative responses are linear; that is, each addition of an element to the memory set causes an identical increment in response time (Sternberg, 1966). This has led him to a theory of the search process in which three components may be identified. During the first component, $S$ processes the test stimulus, transforming it into the form used for subsequent comparisons with the memory set. During the second component, $\mathbf{S}$ searches through short-term memory, comparing the test stimulus representation to the memory set that has been stored there. The third component occurs when $S$ makes his response, based on the results of his search of short-term memory. If this search has been successful (i.e., if he has matched the test stimulus with one of the elements in the memory set), he makes a positive response. Otherwise, his response is negative. The slope of the reaction-time function for each type of response is interpreted as a measure of the time used for the second component of this three-part process, while the intercept of the function measures both the time used for preprocessing the test stimulus and the time necessary for responding once the answer in known.

Another important finding concerns the nature of the comparison task. Two hypotheses about this task have been presented: exhaustive search and self-terminating search. An exhaustive search means tilat $S$ compares every member of the memory set to the test stimulus before making a response, regardless of whether or not a match has been made. In contrast, a self-terminating search is one in which only as many comparisons as necessary are made; that is, $S$ responds as soon as a match is made in the case of a positive response and searches the entire set in the case of a negative response. In the exhaustive case, the same number of comparisons are made for both a positive and negative response, so the slopes of the reaction-time functions for the two responses should be equal. In the self-terminating case, on the other hand, $S$ must search, on the average, only half the memory set before making a positive response, whereas he must search the entire set before making a negative response. This leads to the prediction that the slope of the function for a positive response will be half the slope for a negative response. In particular, reaction time (RT) for the exhaustive case is $\mathrm{RT}(\mathrm{d})=\left(\pi+\rho_{\mathrm{y}}\right)+\mathrm{d} \kappa$ for a positive response; $\operatorname{RT}(\mathrm{d})=\left(\pi+\rho_{\mathrm{n}}\right)+\mathrm{d} \kappa$ for $\mathrm{a}$ negative response. For the self-terminating case, $\operatorname{RT}(\mathrm{d})=\left(\pi+\rho_{\mathrm{y}}\right)+1 / 2(\mathrm{~d}+1) \kappa$ for a positive response and response. In the above equations, $d=$ size of memory set, $\pi=$ time for processing the test stimulus, and $k=$ time for a single comparison; $\rho=$ response time, and the subscripts $\mathbf{y}$ and $\mathbf{n}$ denote positive and negative responses, respectively. Sternberg (1966) has found that the slopes for the positive and negative functions are equal, which supports the exhaustive search hypothesis. He has also found that the intercept for negative responses is usually 40 or $50 \mathrm{msec}$ greater than the intercept for positive responses.

Search experiments of the Sternberg type have usually used the same symbols for test stimulus and memory set elements. The present experiment, in contrast, was concerned with the case in which the test stimulus was not directly comparable to the memory set. While members of the memory sets were letters of the alphabet, the test stimulus on any trial could be a letter, a word, or a picture. If the test stimulus was a letter, $S$ made a positive response only if that letter was a member of the memory set. This is precisely the task used by Sternberg for digit stimuli (1966). If the test stimulus was a word, S made a positive response only if the first letter of that word was a member of the memory set. Finally, if the test stimulus was a picture, $S$ made a positive response only if the first letter of the name of that picture was a member of the memory set.

In this experiment, the search paradigm involved retrieval of information from long-term memory when the picture stimuli were used. If the test stimulus was a picture, a direct comparison with the memory set was impossible. Instead, the picture test stimulus served to instigate a search of long-term memory, a search essential to obtaining a symbol comparable to the representation of the memory set stored in short-term memory. It was assumed that given a picture stimulus, $S$ had to retrieve the name of that picture from long-term memory, translate that name to its first letter, and then transform the letter into a form that could be compared to the memory set representation before searching for a match. If the test stimulus was a letter, the first two steps of this process were unnecessary. In this case, $S$ could immediately begin transforming the test stimulus into the form used for comparisons. Finally, word stimuli were 
used to represent an intermediate stage in the search process. Given a word stimulus, $S$ had to extract the first letter of the word and then transform it before beginning his search of short-term memory. One might hypothesize that the time necessary to translate the retrieved name of a picture stimulus into a representation of its first letter would correspond to the time necessary to change a word stimulus in to a similar representation, although this is a rather tenuous hypothesis.

The data of interest in this study are the slopes and intercepts of the reaction-time functions for each type of test stimulus and each type of response. The form of these functions may be used to evaluate several hypotheses. For example, if the stimulus representations ultimately compared to the memory set were the same for each type of test stimulus, there should be no differences among these stimuli in the time required for a single comparison $(\kappa)$. This leads to the prediction that for both positive and negative responses, the slopes of the reaction-time functions for letters, words, and pictures should be identical. If these slopes were not identical, a difference in comparison time and a corresponding difference in the representations of the test stimulus would be implied. Intercept differences among the reaction-time functions for letters, words, and pictures may be interpreted as representing differences in initial processing time $(\pi)$, response time $(\rho)$, or both. If the time required to obtain a representation of the picture stimulus comparable to the representation of the memory set in short-term memory includes both the processing time normally required for letter stimuli and the time required to retrieve the name of the picture from long-term memory, then the intercepts of the reaction-time functions for letters should be less than the intercepts for pictures. However, such intercept differences could also be interpreted in terms of different response times $(\rho)$ for the two types of stimuli. (Moreover, in the case of a self-terminating search, the intercept of the function for a negative response includes the term $1 / 2 k$, so differences in comparison times would contribute to differences in the intercept of that function.) Of course, both slope and intercept differences could be present, indicating that both comparison time and precomparison processing or response procedures are not the same for the two types of stimuli. Finally, a comparison of the slopes of the reaction-time functions for positive vs negative responses for each type of stimulus serves to indicate whether the search process for that stimulus is exhaustive or self-terminating. It is possible, of course, that the process used could vary with the type of test stimulus. This experiment was conducted in order to test these various predictions.

\section{Subjects \\ METHOD}

The Ss were 10 girls who were in Grades 11 or 12 in high schools in the vicinity of Stanford University. They were paid $\$ 1.75$ for each of the five experimental sessions.

\section{Stimuli}

The memory sets consisted of two or four capital letters typed with an IBM Executive Registry electric typewriter and separated by a single space. No letter was duplicated within a memory set. Each set was displayed on a $5 \times 8$ in. white file card, with the center of the display located at the center of S's visual area. The set of letters whose elements were used will be called the letter set; this consisted of all letters except the five vowels and V, X, and Y.

Eighteen letters, 18 words, and 18 pictures were used as test stimuli. Each test stimulus was displayed on a $5 \times 8$ in. white file card with the center of the stimulus at the center of S's viewing area. The letter stimuli consisted of single members of the letter set, one corresponding to each letter set element. Each letter was typed in capitalized form with the same typewriter as that used for memory set displays. To each letter stimulus, there corresponded a word test stimulus beginning with that letter. These word stimuli were common nouns (e.g., $s=$ snake, $w=$ whistle, etc.) whose length ranged from three to seven letters: Since the center of the word coincided with the center of S's visual area, the position of the first letter relative to the center varied. The words were typed in capitals with the same typewriter used for the memory sets and letter stimuli. Picture test stimuli were black-and-white drawings, copies of illustrations used in The Golden Happy Book of ABC (Golden Press), a children's picture book. The height and width of these pictures varied from $11 / 4 \mathrm{in}$. to $31 / 4 \mathrm{in}$. and $13 / 8 \mathrm{in}$. to 4 in., respectively. Each picture represented one of the common nouns used as word stimuli.

\section{Apparatus}

The apparatus consisted of an Iconix tachistoscope and exposure box (System 153). The visual area exposed to $S$ measured $7 \times 31 / 2$ in., and the viewing distance was approximately $2 \mathrm{ft}$. Between stimulus exposures, the viewing area was illuminated by a light of $1.4 \mathrm{fL}$, while the display brightness averaged $39 \mathrm{fL}$. A black dot marked the center of the pre- and postexposure field.

On a table to the right of $S$, three telegraph keys were placed in an arc, separated by a distance of $4.3 \mathrm{~cm}$. The $S$ rested her right arm on the table and depressed the keys with both her forefinger and second finger; $S$ reports from previous experiments have indicated that the use of two fingers makes the response easier and more natural. Half of the Ss were randomly chosen to depress the key on the right for a positive response and the key on the left for a negative response; for the remainder, these conditions were reversed. The $S$ was instructed to depress the center key until she made her response; this procedure prevented her from biasing her response by maintaining a hand position closer to one of the keys than the other. A light on the control panel monitored by $E$ enabled her to ensure that $S$ was following this instruction.

Procedure

Each $S$ participated in five sessions of 54 trials. At the beginning of each session, $S$ viewed and named each member of the set of picture stimuli before beginning the series of trials.

Each memory șet size ( 2 or 4 ), each response (positive or negative), and each serial position for a correct response was used equally often in a session. In addition, letter, word, and picture test stimuli were used equally often for each set size, response, and position. Within these limits, the order of presentation of trials and the particular stimuli used were randomized.

Each trial lasted approximately $15 \mathrm{sec}$ and involved the following sequence of events:

1. S depressed the center key and held it down.

2. E exposed the memory set for study until $S$ verbally reported that she was ready.

3. E removed the memory set, inserted the test display, and verbally informed $S$ that she had done so. (This procedure lasted about $3 \mathrm{sec}$.)

4. $S$ pushed a button held in her left hand, and after a .4sec delay, the test stimulus was exposed for $400 \mathrm{msec}$. The onset of the stimulus exposure coincided with the onset of a latency counter.

5. Using her right hand, $S$ made the appropriate response by releasing the center key and depressing the key to the right or left. This response stopped the latency counter.

6. S reported the particular letter, word, or picture that had been used as the test stimulus.

7. E told $S$ if she had made an error.

\section{RESULTS}

The mean latencies in msec for the 


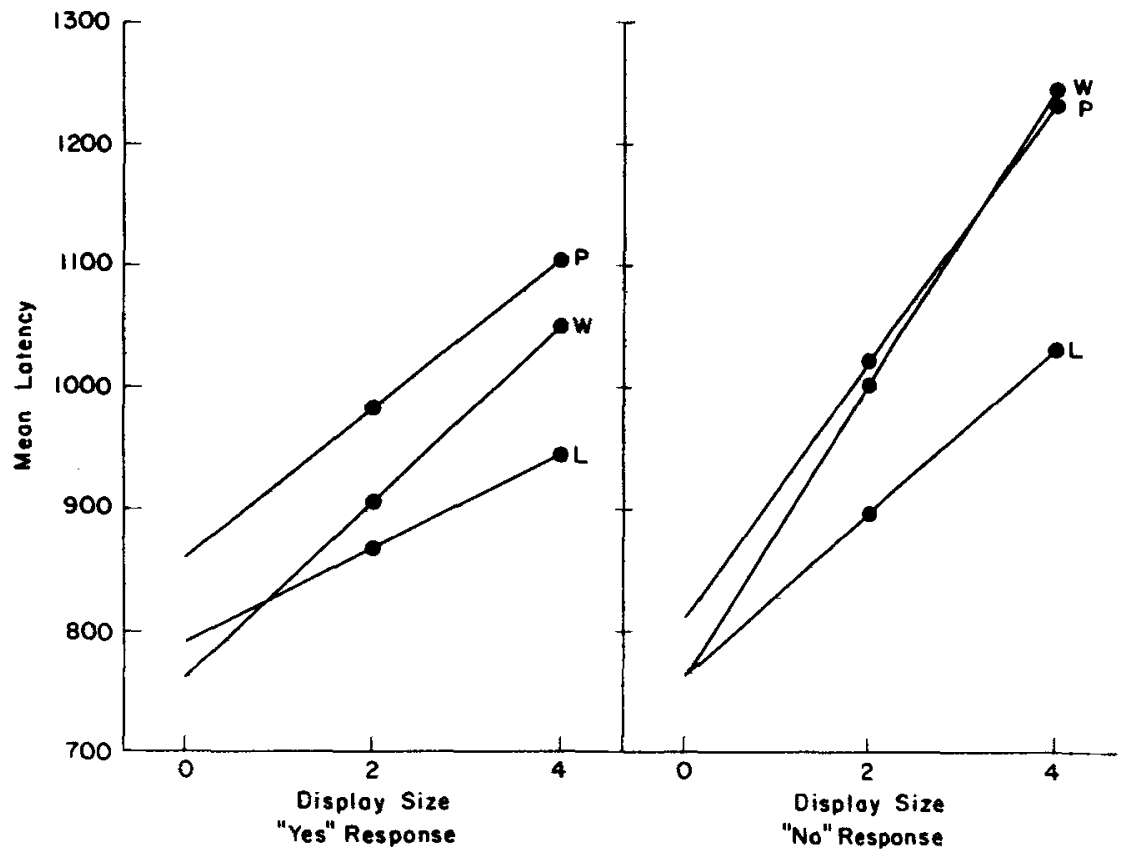

group of $10 \mathrm{Ss}$ are presented in Fig. 1. The error rate for each $S$ was low (with a mean of $2.2 \%$ and a range over Ss from $.5 \%$ to $5 \%$ ), and all analyses are based only on the data for correct responses. The data from the first session were discarded in order to allow for the possibility that $S$ had not fully habituated to the task. In addition, the first six trials of subsequent sessions were regarded as warm-up trials, and omitted from the analysis. Although an improvement in mean performance over sessions was found, when data for Sessions 2 and 3 were compared to data for the last

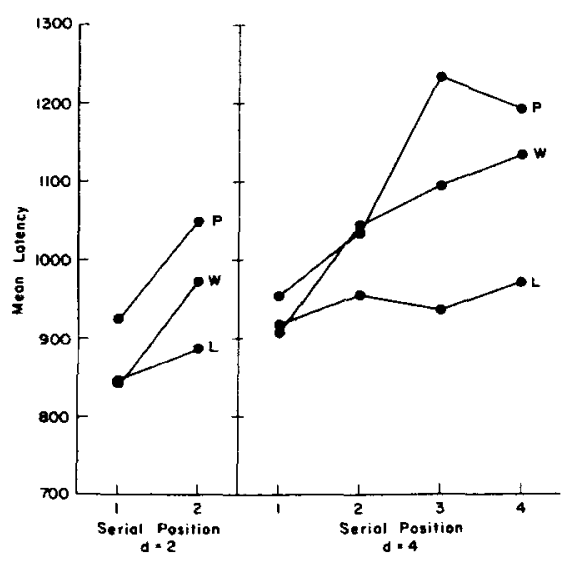

Fig. 2. Latency in msec of positive responses as a function of the serial position of the test stimulus in the memory set for sets of two (left panel) or four (right panel) elements, averaged over Ss and Sessions 2 to 5 . ( $L=$ letters, $W=$ words, and $P=$ pictures.) negative response, while for Ss 6-10, the situation was reversed.) Table 1 presents
Fig. 1. Latency in msec of positive (left panel) and negative (right panel) responses to each type of stimulus as a function of the size of the memory set, averaged over Ss and Sessions 2 to 5 . ( $L=$ letters, $W=$ words, and $P=$ pictures.)

the mean latency over days for each type of stimulus and each response. In Table 2, the slopes and intercepts of the reaction-time functions for each $S$ and for the mean over Ss are given. In addition, this table shows the ratio of the slope of the function for negative responses to the slope for positive responses. (For the mean over Ss, this ratio corresponds not to the average of the individual slope ratios, but to the ratio of the average slopes.) For a given function, the value of the slope given in the table was calculated by the formula: Slope $=[R T(4)-R T(2)] / 2$. In terms of the equations we have presented, the slope value represents $k$ in the case of negative responses, while for positive responses it represents $\kappa$ or $1 / 2 k$ for exhaustive or self-terminating hypotheses respectively. The ratio of the slope for negative responses to the slope for positive responses thus represents either $\kappa / \kappa$ (for the exhaustive case) or $\kappa / 1 / 2 \kappa$ (for the self-terminating case). If the search process were exhaustive, this ratio should be 1.0 , while a self-terminating search would imply a ratio of 2.0 . Finally, the intercepts given in Table 2 were calculated by the formula: Intercept $=R T(d)-(d x$ slope $)$. For positive responses, these intercepts represent the value $\left(\pi+\rho_{y}\right)$. For negative responses, they represent $\left(\pi+\rho_{n}\right)$ or $\left(\pi+\rho_{n}+1 / 2 \kappa\right)$ for the exhaustive case or self-terminating case, respectively.

\section{DISCUSSION}

Using digit stimuli, Sternberg (1966)

Table 1

Mean Latencies in Msec Over Sessions 2 to $S$ for Each $S$ and for the Group; d Denotes the Size of the Memory Set

\begin{tabular}{|c|c|c|c|c|c|c|c|c|c|c|c|c|}
\hline \multirow{3}{*}{$\begin{array}{c}\text { S } \\
\text { No. }\end{array}$} & \multicolumn{4}{|c|}{ Letter } & \multicolumn{4}{|c|}{ Word } & \multicolumn{4}{|c|}{ Picture } \\
\hline & \multicolumn{2}{|c|}{$\mathrm{d}=2$} & \multicolumn{2}{|c|}{$\mathrm{d}=4$} & \multicolumn{2}{|c|}{$d=2$} & \multicolumn{2}{|c|}{$d=4$} & \multicolumn{2}{|c|}{$d=2$} & \multicolumn{2}{|c|}{$\mathrm{d}=4$} \\
\hline & Yes & No & Yes & No & Yes & No & Yes & No & Yes & No & Yes & No \\
\hline 1 & 788 & 827 & 828 & 845 & 832 & 920 & 932 & 1109 & 844 & 910 & 957 & 1042 \\
\hline 2 & 1046 & 1138 & 1062 & 1144 & 1124 & 1257 & 1171 & 1305 & 1208 & 1209 & 1254 & 1278 \\
\hline 3 & 819 & 793 & 894 & 993 & 826 & 865 & 999 & 1234 & 910 & 907 & 1100 & 1307 \\
\hline 4 & 879 & 866 & 892 & 895 & 860 & 908 & 980 & 1022 & 930 & 917 & 1027 & 992 \\
\hline 5 & 770 & 737 & 927 & 946 & 836 & 940 & 1159 & 1108 & 916 & 1032 & 1104 & 1165 \\
\hline 6 & 781 & 892 & 873 & 1069 & 853 & 995 & 1005 & 1365 & 952 & 960 & 957 & 1118 \\
\hline 7 & 730 & 801 & 800 & 958 & 853 & 928 & 870 & 1124 & 922 & 922 & 1002 & 1172 \\
\hline 8 & 1242 & 1253 & 1366 & 1644 & 1262 & 1422 & 1505 & 2064 & 1350 & 1538 & 1568 & 2106 \\
\hline 9 & 659 & 702 & 733 & 811 & 667 & 717 & 840 & 968 & 805 & 808 & 1008 & 1045 \\
\hline 10 & 976 & 962 & 1066 & 1003 & 972 & 1070 & 1042 & 1166 & 1006 & 1054 & 1074 & 1134 \\
\hline $\begin{array}{l}\text { Group } \\
\text { Mean }\end{array}$ & 869 & 897 & 944 & 1031 & 908 & 1002 & 1050 & 1246 & 984 & 1026 & 1105 & 1236 \\
\hline
\end{tabular}


Table 2

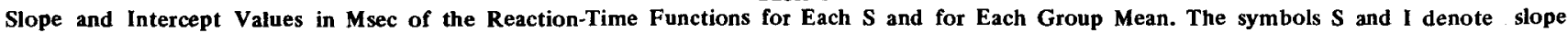
and intercept, respectively; the subscripts $y$ and $n$ denote yes and no responses, respectively.

\begin{tabular}{|c|c|c|c|c|c|c|c|c|c|c|c|c|}
\hline & & 1 & 2 & 3 & 4 & 5 & 6 & 7 & 8 & 9 & 10 & $\begin{array}{l}\text { Mean } \\
\text { All Ss }\end{array}$ \\
\hline 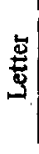 & $\begin{array}{l}S_{y} \\
I_{y} \\
S_{n} \\
I_{n} \\
S_{n} / S_{y}\end{array}$ & $\begin{array}{c}20.0 \\
748 \\
9.0 \\
809 \\
.45\end{array}$ & $\begin{array}{c}8.0 \\
1030 \\
3.0 \\
1132 \\
.38\end{array}$ & $\begin{array}{l}37.5 \\
744 \\
100.0 \\
593 \\
2.67\end{array}$ & $\begin{array}{c}6.5 \\
866 \\
14.5 \\
837 \\
2.23\end{array}$ & $\begin{array}{l}78.5 \\
613 \\
104.5 \\
528 \\
\quad 1.33\end{array}$ & $\begin{array}{c}46.0 \\
689 \\
88.5 \\
715 \\
1.92\end{array}$ & $\begin{array}{c}35.0 \\
660 \\
78.5 \\
644 \\
2.24\end{array}$ & $\begin{array}{c}62.0 \\
1118 \\
195.5 \\
862 \\
3.15\end{array}$ & $\begin{array}{c}37.0 \\
585 \\
54.5 \\
593 \\
1.47\end{array}$ & $\begin{array}{c}45.0 \\
886 \\
20.5 \\
921 \\
\quad .46\end{array}$ & $\begin{array}{c}37.5 \\
794 \\
67.0 \\
763 \\
1.79\end{array}$ \\
\hline$\stackrel{?}{2}$ & $\left\{\begin{array}{l}S_{\mathbf{y}} \\
I_{\mathbf{y}} \\
S_{\mathbf{n}} \\
I_{\mathbf{n}} \\
S_{\mathbf{n}} / S_{\mathbf{y}}\end{array}\right.$ & $\begin{array}{c}50.0 \\
732 \\
94.5 \\
731 \\
1.89\end{array}$ & $\begin{array}{c}23.5 \\
1077 \\
24.0 \\
1209 \\
1.02\end{array}$ & $\begin{array}{l}86.5 \\
653 \\
184.5 \\
496 \\
2.13\end{array}$ & $\begin{array}{c}60.0 \\
740 \\
57.0 \\
794 \\
.95\end{array}$ & $\begin{array}{c}161.5 \\
513 \\
84.0 \\
772 \\
.52\end{array}$ & $\begin{array}{l}76.0 \\
701 \\
185.0 \\
625 \\
2.43\end{array}$ & $\begin{array}{c}8.5 \\
836 \\
98.0 \\
732 \\
11.53\end{array}$ & $\begin{array}{c}121.5 \\
1019 \\
321.0 \\
780 \\
2.64\end{array}$ & $\begin{array}{l}86.5 \\
494 \\
125.5 \\
466 \\
\quad 1.45\end{array}$ & $\begin{array}{c}35.0 \\
902 \\
48.0 \\
974 \\
1.37\end{array}$ & $\begin{array}{l}71.0 \\
766 \\
122.0 \\
758 \\
\quad 1.72\end{array}$ \\
\hline 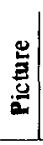 & $\begin{array}{l}S_{y} \\
I_{y} \\
S_{n} \\
I_{n} \\
S_{n} / S_{y}\end{array}$ & $\begin{array}{c}56.5 \\
731 \\
66.0 \\
778 \\
1.17 \\
\end{array}$ & $\begin{array}{c}23.0 \\
1162 \\
34.5 \\
1140 \\
1.50\end{array}$ & $\begin{array}{l}95.0 \\
720 \\
200.0 \\
507 \\
2.10 \\
\end{array}$ & $\begin{array}{c}48.5 \\
833 \\
37.5 \\
842 \\
.77\end{array}$ & $\begin{array}{c}94.0 \\
728 \\
66.5 \\
899 \\
.71 \\
\end{array}$ & $\begin{array}{c}2.5 \\
947 \\
79.0 \\
802 \\
31.60\end{array}$ & $\begin{array}{l}40.0 \\
842 \\
125.0 \\
672 \\
3.12 \\
\end{array}$ & $\begin{array}{l}109.0 \\
1132 \\
284.0 \\
970 \\
2.60 \\
\end{array}$ & $\begin{array}{l}101.5 \\
602 \\
118.5 \\
571 \\
\quad 1.17\end{array}$ & $\begin{array}{c}34.0 \\
938 \\
40.0 \\
974 \\
1.18\end{array}$ & $\begin{array}{l}60.5 \\
863 \\
105.0 \\
816 \\
\quad 1.74\end{array}$ \\
\hline
\end{tabular}

obtained a mean reaction-time function of the form: $R T(d)=397.2+37.9 \mathrm{~d}$. Furthermore, functions obtained for digits and other stimuli (e.g., faces, nonsense forms) all gave qualitatively similar results; that is, linear functions with the same slope for both positive and negative responses, although scanning rates varied somewhat among stimulus materials. On the basis of these findings, similar functions for letter stimuli would be expected, and earlier experiments conducted in our laboratory conform to these expectations. However, in the present experiment, the Sternberg paradigm for letter stimuli was imbedded in a more complex task. That is, when presented with a letter test stimulus, S's task was precisely the same as that required in Sternberg's (1966) experiment, but letter trials were mixed randomly with trials using word and picture stimuli. The resulting data for letter stimuli are qualitatively different from those usually obtained.

In this experiment, both the slopes and intercepts of the reaction-time functions for all three types of test stimuli are larger than those typically found, indicating that the search process is different from that used by $S s$ in the usual Stemberg task. In particular, the increased slope indicates that more time is required for comparisons. One possible explanation for this result is that the representation of the test stimulus that is used for comparisons has changed; this hypothesis is supported by the intercept data, which imply that precomparison processing and/or response time, even for letter stimuli, has also increased. Sternberg (1968) has stated that his results indicate that comparisons are made on the basis of visual rather than acoustic material. For example, the stimulus 8 is compared to members of the memory set in terms of its physical features (e.g., rounded top and bottom) rather than its name, "eight." In our experiment, however, one might suspect that comparisons are made in terms of verbal material. This might occur especially for word and picture stimuli, which require preprocessing of a verbal nature; and it is possible that this verbalization carries over to the case of letter stimuli as well.

Another feature of our data is that the slopes for letter stimuli are less than those for words and pictures. This may indicate that the representation of the stimulus which is used for comparison with the memory set in the case of letters is different from that used for word and picture stimuli. If the representations are identical for all three types of stimuli, the reaction-time functions should be parallel, with the lowest intercept corresponding to letters. This would imply that the only difference in the search process for the three stimulus types is the amount of preprocessing conducted. However, this is not the case. On the other hand, the lower slope for letters could result from the mixing of two different search strategies, one exhaustive and one self-terminating, a possibility considered in more detail below.

In addition, we found that equality of slopes for positive and negative responses does not hold in this experiment. The ratio of the slope for negative responses to the slope for positive responses is approximately 1.75 for all three types of stimuli instead of 1.0 as found by Sternberg. This implies that at least some Ss depart from an exhaustive strategy, even in the case of letter stimuli.

The serial position curves also indicate that Ss may not be searching exhaustively. In an exhaustive search, all members of the memory set are compared to the test stimulus, with the result that reaction time is independent of the position of the test stimulus in the set. Thus, serial position curves should be flat if an exhaustive search is used. Flat serial position curves would also be found in the case of a self-terminating search if either the order or the starting point of comparisons were random, since the search would require on the average an equal amount of time for each serial position. However, if comparisons always began with the leftmost member of the memory set and proceeded from left to right, a self-terminating search would result in linearly increasing serial position curves. In Fig. 2 , the serial position curves are increasing, which gives additional support to the hypothesis that self-terminating searches are occurring.

Sternberg (1968) has proposed a model which attempts to explain why exhaustive scans occur. According to his model, a "homonculus" operates a scanner, which delivers material for comparison to a comparator, and also examines a match register to which the comparator sends a signal if a match is made. Since the homonculus cannot perform both of these functions simultaneously (and switching from one function to the other takes time), it acts so as to minimize the total time involved in the search process. If it takes longer to make a check of the match register after each comparison than to check the register only once, after all comparisons, the homonculus operates exhaustively. On the other hand, if the time required for a single scan and comparison is large relative to the time required to switch functions and check the match register, a self-terminating process is more efficient. In fact, Sternberg (1967a) has found that self-terminating scans occur when the comparison rate is slow (approximately $124 \mathrm{msec}$ ).

In the present case, comparison time $(K)$ is much greater than in Sternberg's (1966) experiment, perhaps because the basis for comparison is no longer visual. Thus, a 
self-terminating search might be advantageous. Moreover, if this self-terminating process were carried over to the case of letter stimuli for a portion but not all of the trials, this would account for the fact that the slope of the reaction-time function for letters is less than that for words and pictures. It would also explain why the letter slope for negative responses is approximately twice that found by Sternberg for digits. (The slope for negative responses gives a more accurate estimate of comparison time because it covers the case in which a comparison is made for each memory set stimulus.) Possibly, comparisons of letter stimuli are sometimes based on visual representations and sometimes on verbal representations, and the search process is varied accordingly.

In an attempt to clarify the results of this experiment, another study is now being conducted. In this study, word stimuli have been eliminated, and the test stimuli for a given session may be pictures only, letters only, or a mixture of pictures and letters. For sessions that involve only letters as test stimuli, the task is the same as that used by Sternberg (1966) with digits, and on the basis of previous work in our laboratory, a replication of his results is expected. On the other hand, the results of picture only and mixed picture and letter sessions should be like those obtained in the present experiment. Moreover, additional memory set sizes are being used in the study currently in progress. Because the data obtained in the first experiment are very different from the usual results obtained in the Sternberg task, there is a possibility that the reaction-time functions depart from linearity, and the use of several values of $d$ will indicate whether or not these functions are in fact linear. Hopefully, as a result of these changes, this second experiment will serve to present a better picture of search processes which involve in part a retrieval of information from long-term memory.

\section{REFERENCES}

STERNBERG, S. High speed scanning in human memory. Science, 1966, 153, 652-654.

STERNBERG, S. Retrieval of contextual information from memory. Psychonomic Science, $1967 \mathrm{a}, 8,55-56$.

STERNBERG, $S$. Two operations in character-recognition: Some evidence from reaction-time measurements. Perception \& Psychophysics, 1967b, 2, 45-53.

STERNBERG, S. Inferring the organization of cognitive events from reaction-time: Some scanning processes in active memory. Invited paper at the Symposium on Research in Cognitive Psychology held to inaugurate the Center for Research in Cognition and Affect of the City University of New York, June 7, 1968.

\section{NOTES}

1. This research was supported by Grant NGR 05-020-244 from the National Aeronautics and Space Administration.

2. Address: Department of Psychology, Stanford University, Stanford, California 94305 . (Accepted for publication November 17, 1969.) 